Stud. Univ. Babeş-Bolyai Math. 64(2019), No. 3, 313-329

DOI: http://dx.doi.org/10.24193/subbmath.2019.3.03

\title{
Approximation theorems for multivariate Taylor-Abel-Poisson means
}

\author{
Jürgen Prestin, Viktor Savchuk and Andrii Shidlich
}

Dedicated to Professor Heiner Gonska on the occasion of his 70th anniversary.

\begin{abstract}
We obtain direct and inverse approximation theorems of functions of several variables by Taylor-Abel-Poisson means in the integral metrics. We also show that norms of multipliers in the spaces $L_{p, Y}\left(\mathbb{T}^{d}\right)$ are equivalent for all positive integers $d$.
\end{abstract}

Mathematics Subject Classification (2010): 41A27, 42A45, 41A35.

Keywords: Direct approximation theorem, inverse approximation theorem, Taylor-Abel-Poisson means, $K$-functional, multiplier.

\section{Introduction}

It is well-known that any function $f \in L_{p}\left(\mathbb{T}^{1}\right)$ that is different from a constant can be approximated by its Abel-Poisson means $f(\varrho, \cdot)$ with a precision not better than $1-\varrho$. It relates to the so-called saturation property of this approximation method. From this property, it follows that for any $f \in L_{p}\left(\mathbb{T}^{1}\right)$, the relation

$$
\|f-f(\varrho, \cdot)\|_{p}=\mathcal{O}(1-\varrho), \varrho \rightarrow 1-,
$$

only holds in the trivial case when $f$ is a constant function. Therefore, any additional restrictions on the smoothness of functions do not give us any order of approximation better than $1-\varrho$. In this connection, a natural question is to find a linear operator, constructed similarly to the Poisson operator, which takes into account the smoothness properties of functions and at the same time, for a given functional class, is the best in a certain sense. In [19], for classes of convolutions whose kernels were generated by some moment sequences, the authors proposed a general method of construction of similar operators that take into account properties of such kernels and hence, the smoothness of functions from corresponding classes. One example of such operators are the operators $A_{\varrho, r}$, which are the main subject of study in this paper. 
The operators $A_{\varrho, r}$ were first studied in [15] where, in the terms of these operators, the author gave the structural characteristic of Hardy-Lipschitz classes $H_{p}^{r} \operatorname{Lip} \alpha$ of functions of one variable, holomorphic on the unit disc of the complex plane. In [17], in terms of approximation estimates of such operators in some spaces $S^{p}$ of Sobolev type, the authors give a constructive description of classes of functions of several variables whose generalized derivatives belong to the classes $S^{p} H_{\omega}$. In [13], direct and inverse approximation theorems of $2 \pi$-periodic functions by the operators $A_{\varrho, r}$ were given in the terms of $K$-functionals of functions generated by their radial derivatives.

Approximations of functions of one variable by similar operators of polynomial type were studied in [11], [4], [7], [10], [12], [6] etc. In particular, in [7], the authors found the degree of convergence of the well-known Euler and Taylor means to the functions $f$ from some subclasses of the Lipschitz classes Lip $\alpha$ in the uniform norm. In [12], the analogous results for Taylor means were obtained in the $L_{p}$-norm.

In the present paper, we continue the study of approximative properties of the operators $A_{\varrho, r}$. In particular, we extend the results of the paper [13] to the multivariate case and prove direct and inverse approximation theorems of functions of several variables by the operators $A_{\varrho, r}$ in the integral metrics. We also show that norms of multipliers in the spaces $L_{p, Y}\left(\mathbb{T}^{d}\right)$ are equivalent for all positive integers $d$.

\section{Preliminaries}

Let $d$ be an integer, let $\mathbb{R}^{d}, \mathbb{R}_{+}^{d}$ and $\mathbb{Z}^{d}$ be the sets of all vectors $\mathbf{k}:=\left(k_{1}, \ldots, k_{d}\right)$ with real, real non-negative and integer coordinates respectively. Set $\mathbb{T}^{d}:=\mathbb{R}^{d} / 2 \pi \mathbb{Z}^{d}$. Further, let $L_{p}\left(\mathbb{T}^{d}\right), 1 \leq p \leq \infty$, be the space of all functions $f(\mathbf{x})=f\left(x_{1}, \ldots, x_{d}\right)$ defined on $\mathbb{R}^{d}, 2 \pi$-periodic in each variable with the finite norm

$$
\|f\|_{p}=\|f\|_{L_{p}\left(\mathbb{T}^{d}\right)}:=\left\{\begin{array}{lc}
\left(\int_{\mathbb{T}^{d}}|f(\mathbf{x})|^{p} \mathrm{~d} \sigma(\mathbf{x})\right)^{\frac{1}{p}}, & 1 \leq p<\infty, \\
{\operatorname{ess} \sup _{\mathbf{x} \in \mathbb{T}^{d}}|f(\mathbf{x})|,} & p=\infty,
\end{array}\right.
$$

where $\sigma$ is the normalized Lebesgue measure on $\mathbb{T}^{d}$.

Let $(\mathbf{x}, \mathbf{y}):=x_{1} y_{1}+\ldots+x_{d} y_{d}$ denote the inner product of the elements $\mathbf{x}, \mathbf{y} \in \mathbb{R}^{d}$. Let us set $\mathrm{e}_{\mathbf{k}}:=\mathrm{e}_{\mathbf{k}}(\mathbf{x})=\mathrm{e}^{\mathrm{i}(\mathbf{k}, \mathbf{x})}, \mathbf{k} \in \mathbb{Z}^{d}$, and for any function $f \in L_{1}\left(\mathbb{T}^{d}\right)$, define its Fourier coefficients by

$$
\widehat{f}_{\mathbf{k}}:=\int_{\mathbb{T}^{d}} f(\mathbf{x}) \overline{\mathrm{e}}_{\mathbf{k}}(\mathbf{x}) \mathrm{d} \sigma(\mathbf{x}), \quad \mathbf{k} \in \mathbb{Z}^{d},
$$

where $\bar{z}$ is the complex-conjugate number of $z$.

Set $|\mathbf{k}|_{1}:=\sum_{j=1}^{d}\left|k_{j}\right|$, and for any function $f \in L_{1}\left(\mathbb{T}^{d}\right)$ with the Fourier series of the form

$$
S[f](\mathbf{x})=\sum_{\mathbf{k} \in \mathbb{Z}^{d}} \widehat{f}_{\mathbf{k}} \mathrm{e}_{\mathbf{k}}(\mathbf{x})=\sum_{\nu=0}^{\infty} \sum_{|\mathbf{k}|_{1}=\nu} \widehat{f}_{\mathbf{k}} \mathrm{e}_{\mathbf{k}}(\mathbf{x}),
$$

denote by $f(\varrho, \mathbf{x})$ its Poisson integral (the Poisson operator), i.e.,

$$
f(\varrho, \mathbf{x}):=\int_{\mathbb{T}^{d}} f(\mathbf{x}+\mathbf{s}) P(\varrho, \mathbf{s}) \mathrm{d} \sigma(\mathbf{s}),
$$


where $\varrho \in \mathbb{R}_{+}^{d}, \mathbf{x} \in \mathbb{R}^{d}$, the function $P(\varrho, \mathbf{x}):=\sum_{\mathbf{k} \in \mathbb{Z}^{d}} \varrho^{|\mathbf{k}|} \mathrm{e}_{\mathbf{k}}(\mathbf{x})$ is the Poisson kernel,

$$
\varrho^{|\mathbf{k}|}:=\varrho_{1}^{\left|k_{1}\right|} \cdots \varrho_{d}^{\left|k_{d}\right|} .
$$

In what follows, the expression $f(\varrho, \mathbf{x})$ means the Poisson integral, where $\varrho$ is a vector with the same coordinates, i.e., $\varrho=(\varrho, \ldots, \varrho)$. In such case, we have

$$
P(\varrho, \mathbf{x}):=\sum_{\nu=0}^{\infty} \varrho^{\nu} \sum_{|\mathbf{k}|_{1}=\nu} \mathrm{e}_{\mathbf{k}}(\mathbf{x}) .
$$

Let $f \in L_{1}\left(\mathbb{T}^{d}\right)$. For $\varrho \in[0,1)$ and $r \in \mathbb{N}$, we set

$$
A_{\varrho, r}(f)(\mathbf{x}):=\sum_{\nu=0}^{\infty} \lambda_{\nu, r}(\varrho) \sum_{|\mathbf{k}|_{1}=\nu} \widehat{f}_{\mathbf{k}} \mathrm{e}_{\mathbf{k}}(\mathbf{x})
$$

where for $\nu=0,1, \ldots, r-1$, the numbers $\lambda_{\nu, r}(\varrho) \equiv 1$ and for $\nu=r, r+1, \ldots$,

$$
\lambda_{\nu, r}(\varrho):=\sum_{j=0}^{r-1}\left(\begin{array}{l}
\nu \\
j
\end{array}\right)(1-\varrho)^{j} \varrho^{\nu-j}=\sum_{j=0}^{r-1} \frac{(1-\varrho)^{j}}{j !} \frac{d^{j}}{d \varrho^{j}} \varrho^{\nu} .
$$

The transformation $A_{\varrho, r}$ can be considered as a linear operator on $L_{1}\left(\mathbb{T}^{d}\right)$ into itself. Indeed, $\lambda_{\nu, r}(0)=0$ and for all $\nu=r, r+1, \ldots$ and $\varrho \in(0,1)$,

$$
\sum_{j=0}^{r-1}\left(\begin{array}{l}
\nu \\
j
\end{array}\right)(1-\varrho)^{j} \varrho^{\nu-j} \leq r q^{\nu} \nu^{r-1}, \text { where } 0<q:=\max \{1-\varrho, \varrho\}<1 .
$$

Therefore, for any function $f \in L_{1}\left(\mathbb{T}^{d}\right)$ and for any $0<\varrho<1$, the series on the right-hand side of (2.4) is majorized by the convergent series $2 r\|f\|_{1} \sum_{\nu=r}^{\infty} q^{\nu} \nu^{r-1}$.

Leis [11] considered for $f \in L_{p}\left(\mathbb{T}^{1}\right), 1<p<\infty$, the transformation

$$
L_{\varrho, r}(f)(x):=\sum_{k=0}^{r-1} \frac{\mathrm{d}^{k} f(x)}{\mathrm{d} n^{k}} \cdot \frac{(1-\varrho)^{k}}{k !}, \quad r \in \mathbb{N},
$$

where

$$
\frac{\mathrm{d} f(x)}{\mathrm{d} n}=-\left.\frac{\partial f(\varrho, x)}{\partial \varrho}\right|_{\varrho=1}
$$

is the normal derivative of the function $f$. He showed that if $1<p<\infty$ and

$$
\left\|f(\varrho, \cdot)-L_{\varrho, r}(f)(\cdot)\right\|_{p}=\mathcal{O}\left((1-\varrho)^{r} / r !\right), \quad \varrho \rightarrow 1-,
$$

then $\mathrm{d}^{r} f / \mathrm{d} n^{r} \in L_{p}\left(\mathbb{T}^{1}\right)$.

Butzer and Sunouchi [4] considered for $f \in L_{p}\left(\mathbb{T}^{1}\right), 1 \leq p<\infty$, the transformation

$$
B_{\varrho, r}(f)(x):=\sum_{k=0}^{r-1}(-1)^{\frac{k+1}{2}} f^{\{k\}}(x) \frac{(-\ln \varrho)^{k}}{k !},
$$


where $f^{\{k\}}:=f^{(k)}$ for $k \in 2 \mathbb{Z}_{+}$and $f^{\{k\}}:=\widetilde{f}^{(k)}$ for $k-1 \in 2 \mathbb{Z}_{+}$, where

$$
\widetilde{f}(x)=\lim _{\varepsilon \rightarrow 0}-\frac{1}{\pi} \int_{\varepsilon}^{\pi}(f(x+u)-f(x-u)) \frac{1}{2} \cot \frac{u}{2} \mathrm{~d} u .
$$

They proved the following theorem:

Theorem A. [4]. Assume that $f \in L_{p}\left(\mathbb{T}^{1}\right), 1 \leq p<\infty$.

i) If the derivatives $f^{\{j\}}, j=0,1, \ldots, r-1$, are absolutely continuous and $f^{\{r\}} \in$ $L_{p}\left(\mathbb{T}^{1}\right)$, then

$$
\left\|f(\varrho, \cdot)-B_{\varrho, r}(f)(\cdot)\right\|_{p}=\mathcal{O}\left((-\ln \varrho)^{r} / r !\right), \quad \varrho \rightarrow 1-.
$$

ii) If the derivatives $f^{\{j\}}, j=0,1, \ldots, r-2, r \geq 2$, are absolutely continuous, $f^{\{r-1\}} \in L_{p}\left(\mathbb{T}^{1}\right), 1<p<\infty$, and relation (2.6) holds, then $\tilde{f}^{\{r-1\}}$ is absolutely continuous and $\widetilde{f}\{r\} \in L_{p}\left(\mathbb{T}^{1}\right)$.

These results summarize the approximation behaviour of the operators $L_{\varrho, r}$ and $B_{\varrho, r}$ in the space $L_{p}\left(\mathbb{T}^{1}\right)$. In particular, Leis's result and the statement $i i$ ) of Theorem A represent the so-called inverse theorems and the statement $i$ ) is the so-called direct theorem. Direct and inverse theorems are among the main theorems of approximation theory. They were studied by many authors. Here, we mention only the books [3], [8], [18] which contain fundamental results in this subject. The result of Leis and Theorem A are based on the investigations in the papers [5], [2], where the authors find the direct and inverse approximation theorems for the one-parameter semi-groups of bounded linear transformations $\{T(t)\}$ of some Banach space $X$ into itself by the "Taylor polynomial" $\sum_{k=0}^{r-1}\left(t^{k} / k !\right) A^{k} f$, where $A f$ is the infinitesimal operator of a semi-group $\{T(t)\}$.

The transformations $A_{\varrho, r}$ considered in this paper are similar to the transformations $L_{\varrho, r}$ and $B_{\varrho, r}$ as they are also based on the "Taylor polynomials". The relation between the operators $A_{\varrho, r}$ and the "Taylor polynomials" is shown in the following statement.

Lemma 2.1. Assume that $f \in L_{1}\left(\mathbb{T}^{d}\right)$. Then for any numbers $r \in \mathbb{N}, \varrho \in[0,1)$ and $\mathbf{x} \in \mathbb{T}^{d}$,

$$
A_{\varrho, r}(f)(\mathbf{x})=\sum_{j=0}^{r-1} \frac{\partial^{j} f(\varrho, \mathbf{x})}{\partial \varrho^{j}} \cdot \frac{(1-\varrho)^{j}}{j !} .
$$

Proof. With respect to the variable $\varrho$, let us differentiate the decomposition of the Poisson integral into the uniformly convergent series

$$
f(\varrho, \mathbf{x})=\sum_{\nu=0}^{\infty} \varrho^{\nu} \sum_{|\mathbf{k}|_{1}=\nu} \widehat{f}_{\mathbf{k}} \mathrm{e}_{\mathbf{k}}(\mathbf{x}), \quad \varrho \in[0,1), \mathbf{x} \in \mathbb{T}^{d}
$$

We see that for any $j=0,1, \ldots$

$$
\frac{\partial^{j} f(\varrho, \mathbf{x})}{\partial \varrho^{j}}=\sum_{\nu=j}^{\infty} \frac{\nu !}{(\nu-j) !} \varrho^{\nu-j} \sum_{|\mathbf{k}|_{1}=\nu} \widehat{f}_{\mathbf{k}} \mathrm{e}_{\mathbf{k}}(\mathbf{x}) .
$$


Since

$$
\sum_{j=0}^{\nu}\left(\begin{array}{l}
\nu \\
j
\end{array}\right)(1-\varrho)^{j} \varrho^{\nu-j}=((1-\varrho)+\varrho)^{\nu}=1, \nu=0,1, \ldots
$$

then

$$
\begin{gathered}
\sum_{j=0}^{r-1} \frac{\partial^{j} f(\varrho, \mathbf{x})}{\partial \varrho^{j}} \cdot \frac{(1-\varrho)^{j}}{j !}=\sum_{\nu=0}^{r-1} \sum_{j=0}^{\nu}\left(\begin{array}{l}
\nu \\
j
\end{array}\right)(1-\varrho)^{j} \varrho^{\nu-j} \sum_{|\mathbf{k}|_{1}=\nu} \widehat{f}_{\mathbf{k}} \mathrm{e}_{\mathbf{k}}(\mathbf{x}) \\
+\sum_{\nu=r}^{\infty} \sum_{j=0}^{r-1}\left(\begin{array}{l}
\nu \\
j
\end{array}\right)(1-\varrho)^{j} \varrho^{\nu-j} \sum_{|\mathbf{k}|_{1}=\nu} \widehat{f}_{\mathbf{k}} \mathrm{e}_{\mathbf{k}}(\mathbf{x})=A_{\varrho, r}(f)(\mathbf{x}) .
\end{gathered}
$$

\section{Direct and inverse approximation theorems}

\subsection{Radial derivatives and $K$-functionals}

If for a function $f \in L_{1}\left(\mathbb{T}^{d}\right)$ and for a positive integer $n$ there exists the function $g \in L_{1}\left(\mathbb{T}^{d}\right)$ such that

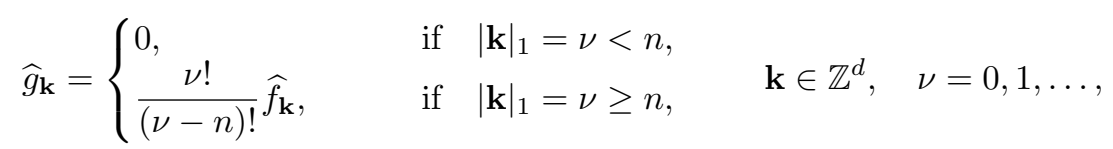

then we say that for the function $f$, there exists the radial derivative $g$ of order $n$ for which we use the notation $f^{[n]}$.

Let us note that if the function $f^{[r]} \in L_{1}\left(\mathbb{T}^{d}\right)$, then its Poisson integral can be presented as

$$
f^{[r]}(\varrho, \mathbf{x})=(f(\varrho, \cdot))^{[r]}(\mathbf{x})=\varrho^{r} \frac{\partial^{r} f(\varrho, \mathbf{x})}{\partial \varrho^{r}} \quad \varrho \in[0,1), \forall \mathbf{x} \in \mathbb{T}^{d} .
$$

In the space $L_{p}\left(\mathbb{T}^{d}\right)$, the $K$-functional of a function $f$ (see, for example, [8, Chap. 6]) generated by the radial derivative of order $n$ is the following quantity:

$$
K_{n}(\delta, f)_{p}:=\inf \left\{\|f-h\|_{p}+\delta^{n}\left\|h^{[n]}\right\|_{p}: h^{[n]} \in L_{p}\left(\mathbb{T}^{d}\right)\right\}, \quad \delta>0 .
$$

\subsection{Main results}

Let $\mathbb{Z}_{-}^{d}$ denote the set of all vectors $\mathbf{k}:=\left(k_{1}, \ldots, k_{d}\right)$ with negative integer coordinates, $\mathbb{Z}_{+}^{d}:=\mathbb{Z}^{d} \cap \mathbb{R}_{+}^{d}$ and $Y:=\mathbb{Z}_{+}^{d} \cup \mathbb{Z}_{-}^{d}$. Let also $L_{p, Y}\left(\mathbb{T}^{d}\right)$ be the set of all functions $f$ from $L_{p}\left(\mathbb{T}^{d}\right)$ such that the Fourier coefficients $\widehat{f}_{\mathbf{k}}=0$ for all $\mathbf{k} \in \mathbb{Z}^{d} \backslash Y$. Further, we consider the functions $\omega(t), t \in[0,1]$, satisfying the following conditions 1)-4): 1) $\omega(t)$ is continuous on $[0,1]$; 2) $\omega(t)$ is monotonically increasing; 3) $\omega(t) \neq 0$ for all $t \in(0,1]$; 4) $\omega(t) \rightarrow 0$ as $t \rightarrow 0$; and the well-known Zygmund-Bari-Stechkin conditions (see, for example, [1]):

$$
(\mathcal{Z}): \int_{0}^{\delta} \frac{\omega(t)}{t} \mathrm{~d} t=\mathcal{O}(\omega(\delta)), \quad\left(\mathcal{Z}_{n}\right): \int_{\delta}^{1} \frac{\omega(t)}{t^{n+1}} \mathrm{~d} t=\mathcal{O}\left(\frac{\omega(\delta)}{\delta^{n}}\right), n \in \mathbb{N}, \delta \rightarrow 0+.
$$

The main results of this paper are contained in the following two statements: 
Theorem 3.1. Assume that $f \in L_{p, Y}\left(\mathbb{T}^{d}\right), 1 \leq p \leq \infty, n, r \in \mathbb{N}, n \leq r$ and the function $\omega(t), t \in[0,1]$, satisfies conditions 1)-4) and $(\mathcal{Z})$. If

$$
f^{[r-n]} \in L_{p}\left(\mathbb{T}^{d}\right) \quad \text { and } \quad K_{n}\left(\delta, f^{[r-n]}\right)_{p}=\mathcal{O}(\omega(\delta)), \delta \rightarrow 0+,
$$

then

$$
\left\|f-A_{\varrho, r}(f)\right\|_{p}=\mathcal{O}\left((1-\varrho)^{r-n} \omega(1-\varrho)\right), \quad \varrho \rightarrow 1-
$$

Theorem 3.2. Assume that $f \in L_{p, Y}\left(\mathbb{T}^{d}\right), 1 \leq p \leq \infty, n, r \in \mathbb{N}, n \leq r$ and the function $\omega(t), t \in[0,1]$, satisfies conditions 1$)-4),(\mathcal{Z})$ and $\left(\mathcal{Z}_{n}\right)$. If relation (3.3) holds, then relations (3.2) hold as well.

Remark 3.3. For a given $n \in \mathbb{N}$, from condition $\left(\mathcal{Z}_{n}\right)$ it follows that

$$
\liminf _{\delta \rightarrow 0+}\left(\delta^{-n} \omega(\delta)\right)>0
$$

or, equivalently, that

$$
(1-\varrho)^{r-n} \omega(1-\varrho) \gg(1-\varrho)^{r} \quad \text { as } \varrho \rightarrow 1-.
$$

Therefore, if condition $\left(\mathcal{Z}_{n}\right)$ is satisfied, then the quantity on the right-hand side of (3.3) decreases to zero as $\varrho \rightarrow 1-$ not faster than the function $(1-\varrho)^{r}$. Also note that the relation $\left.\left\|f-A_{\varrho, r}(f)\right\|_{p}=\mathcal{O}\left((1-\varrho)^{r}\right)\right), \varrho \rightarrow 1-$, only holds in the trivial case when

$$
f(\mathbf{x})=\sum_{\nu=0}^{n-1} \sum_{|\mathbf{k}|_{1}=\nu} \widehat{f}_{\mathbf{k}} \mathrm{e}_{\mathbf{k}}(\mathbf{x}),
$$

and in such case, the theorems are easily true. This fact is related to the so-called saturation property of the approximation method generated by the operator $A_{\varrho, r}$. In particular, in [15], it was shown that the operator $A_{\varrho, r}$ generates the linear approximation method of holomorphic functions which is saturated in the space $H_{p}$ with the saturation order $(1-\varrho)^{r}$ and the saturation class $H_{p}^{r-1} \operatorname{Lip} 1$.

\subsection{Norms of multipliers in the spaces $L_{p, Y}\left(\mathbb{T}^{d}\right)$}

Before proving Theorems 3.1 and 3.2, let us give some auxiliary results. In particular, the following Lemma 3.4 shows that norms of multipliers in the spaces $L_{p, Y}\left(\mathbb{T}^{d}\right)$ are equivalent for all $d$. In our opinion, such a result is interesting in itself.

Let $\mathrm{M}=\left\{\mu_{\nu}\right\}_{\nu=0}^{\infty}$ be a sequence of arbitrary complex numbers. If, for any function $f \in L_{1, Y}\left(\mathbb{T}^{d}\right)$ with Fourier series of the form (2.2), there exists a function $g \in L_{1, Y}\left(\mathbb{T}^{d}\right)$ with Fourier series of the form

$$
S[g](\mathbf{x})=\sum_{\nu=0}^{\infty} \mu_{\nu} \sum_{\mathbf{k} \in \mathbf{Y}:|\mathbf{k}|_{1}=\nu} \widehat{f}_{\mathbf{k}} \mathrm{e}_{\mathbf{k}}(\mathbf{x}),
$$

then we say that in the space $L_{1, Y}\left(\mathbb{T}^{d}\right)$ the multiplier $\mathrm{M}$ is defined. In this case we use the notation $g=\mathrm{M}(f)$.

Let $B_{p, Y}, 1 \leq p \leq \infty$, be a unit ball of the space $L_{p, Y}\left(\mathbb{T}^{d}\right)$, that is, the set of all functions $f \in L_{p, Y}\left(\mathbb{T}^{d}\right)$ such that $\|f\|_{p} \leq 1$. 
If $\mathrm{M}: L_{p, Y}\left(\mathbb{T}^{d}\right) \rightarrow L_{p, Y}\left(\mathbb{T}^{d}\right)$, then the norm of the operator $\mathrm{M}$ is the number

$$
\|\mathrm{M}\|_{L_{p, Y}\left(\mathbb{T}^{d}\right) \rightarrow L_{p, Y}\left(\mathbb{T}^{d}\right)}=\sup _{f \in B_{p, Y}}\|\mathrm{M}(f)\|_{p}=\sup _{\substack{f \in L_{p, Y}\left(\mathbb{T}^{d}\right), f \neq 0}} \frac{\|\mathrm{M}(f)\|_{p}}{\|f\|_{p}} .
$$

We also denote by $\|\mathrm{M}\|_{L_{p}\left(\mathbb{T}^{1}\right) \rightarrow L_{p}\left(\mathbb{T}^{1}\right)}$ the norm of the operator $\mathrm{M}: L_{p}\left(\mathbb{T}^{1}\right) \rightarrow L_{p}\left(\mathbb{T}^{1}\right)$.

Let us note that if $\mathrm{M}$ is a continues operator from $L_{p, Y}\left(\mathbb{T}^{d}\right)$ to $L_{p, Y}\left(\mathbb{T}^{d}\right)$, then $\mathrm{M}$ is called the multiplier of series of the form $(2.2)$ of $(p, p)$-type (see, for example, $[9$, Ch. 16]).

In [16], the authors proved that the norms of the multipliers $\mathrm{M}$, which are defined in a similar way, for the Hardy spaces $H_{p}\left(\mathbb{D}^{d}\right)$ and $H_{p}\left(\mathbb{D}^{1}\right)$ are equivalent for all $d \in \mathbb{N}$. Without going into the details, we note that the space $H_{p}\left(\mathbb{D}^{d}\right)$ can be considered as the space of all complex-valued functions $f: \mathbb{T}^{d} \rightarrow \mathbb{C}$ such that $|f| \in L_{p}\left(\mathbb{T}^{d}\right)$ and $\widehat{f}(\mathbf{k})=0$ for all $\mathbf{k} \in \mathbb{Z}^{d} \backslash \mathbb{Z}_{+}^{d}$ (see, for example, Theorem 2.1.4 [14]). Here, we complement the result of [16] and show that the norms of the multipliers $\mathrm{M}: L_{p, Y}\left(\mathbb{T}^{d}\right) \rightarrow L_{p, Y}\left(\mathbb{T}^{d}\right)$ are equal as well.

Lemma 3.4. Assume that $1 \leq p \leq \infty, d \in \mathbb{N}$ and $\mathrm{M}$ is a multiplier generated by a sequence of complex numbers $\left\{\mu_{\nu}\right\}_{\nu=0}^{\infty}$. Then

$$
\|\mathrm{M}\|_{L_{p, Y}\left(\mathbb{T}^{d}\right) \rightarrow L_{p, Y}\left(\mathbb{T}^{d}\right)}=\|\mathrm{M}\|_{L_{p}\left(\mathbb{T}^{1}\right) \rightarrow L_{p}\left(\mathbb{T}^{1}\right)} .
$$

Proof. Let $f \in L_{p, Y}\left(\mathbb{T}^{d}\right)$. Note that for almost all $\mathbf{x} \in \mathbb{T}^{d}$, the multiplier $\mathrm{M}$ can be defined by the following rule:

$$
\mathrm{M}(f)(\mathbf{x})=\lim _{\varrho \rightarrow 1-} \mathrm{M}(f)(\varrho, \mathbf{x})
$$

where for $0<\varrho<1$ and $\mathbf{x} \in \mathbb{T}^{d}$,

$$
\mathrm{M}(f)(\varrho, \mathbf{x})=\sum_{\nu=0}^{\infty} \lambda_{\nu} \varrho^{\nu} \sum_{\mathbf{k} \in Y:|\mathbf{k}|_{1}=\nu} \widehat{f}_{\mathbf{k}} \mathrm{e}_{\mathbf{k}}(\mathbf{x}) .
$$

If $f \in L_{p}(\mathbb{T})$, then this rule has the form

$$
\mathrm{M}(f)(\varrho, t)=\lim _{\varrho \rightarrow 1-} \sum_{n \in \mathbb{Z}} \mu_{|n|} \varrho^{|n|} \widehat{f}_{n} \mathrm{e}^{\mathrm{i} n t} .
$$

For any $f \in L_{p, Y}\left(\mathbb{T}^{d}\right)$, we set $\mathrm{M}(f)(\mathbf{z})=\mathrm{M}(f)(\varrho, \mathbf{x})$, where for $0<\varrho_{j}<1$ and $\mathbf{x} \in \mathbb{T}^{d}$, the point $\mathbf{z}:=\left(\varrho_{1} \mathrm{e}^{\mathrm{i} x_{1}}, \ldots, \varrho_{d} \mathrm{e}^{\mathrm{i} x_{d}}\right)$ belongs to the unit polydisc

$$
\mathbb{D}^{d}:=\left\{\mathbf{z} \in \mathbb{C}^{d}: \max _{1 \leq j \leq d}\left|z_{j}\right|<1\right\} .
$$

Therefore, the function $\mathrm{M}(f)(\mathbf{z})$ is a $d$-harmonic function in $\mathbb{D}^{d}$ and according to the assertion (c) of Theorem 2.1.3 [14], we have $\|\mathrm{M}(f)(\varrho \cdot)\|_{p} \leq\|\mathrm{M}(f)\|_{p}$. On the other hand, by virtue of Fatou's lemma,

$$
\|\mathrm{M}(f)\|_{p} \leq \liminf _{\varrho \rightarrow 1-}\|\mathrm{M}(f)(\varrho \cdot)\|_{p},
$$


hence, for $1 \leq p<\infty$,

$$
\|\mathrm{M}(f)\|_{p}=\lim _{\varrho \rightarrow 1-}\|\mathrm{M}(f)(\varrho, \cdot)\|_{p} .
$$

If $p=\infty$, then instead of the last relation we have

$$
\int_{\mathbb{T}^{d}} \mathrm{M}(f)(\mathbf{w}) g(\mathbf{w}) \mathrm{d} \sigma(\mathbf{w})=\lim _{\varrho \rightarrow 1-} \int_{\mathbb{T}^{d}} \mathrm{M}(f)(\varrho, \mathbf{w}) g(\mathbf{w}) \mathrm{d} \sigma(\mathbf{w})
$$

for any function $g \in L_{1}\left(\mathbb{T}^{d}\right)$, i.e., we have convergence in the weak $L_{1}$-topology of space $L_{\infty}\left(\mathbb{T}^{d}\right)$.

Let $f \in L_{p, Y}\left(\mathbb{T}^{d}\right), f \neq 0, \mathbf{z}$ be a fixed point in $\overline{\mathbb{D}}^{d}$ and $0 \leq \varrho<1$. In the disc $\mathbb{D}^{1}$, consider the function $u_{\varrho \mathbf{z}}(\omega):=f(\varrho, \mathbf{z} \omega)$. Applying Lemma 3.3.2 [14], we consistently have the following equality and estimate for the integral of $|\mathrm{M}(f)(\varrho \cdot)|^{p}$ for $0 \leq \varrho<1$ and $1 \leq p<\infty$ :

$$
\begin{aligned}
& \int_{\mathbb{T}^{d}}|\mathrm{M}(f)(\varrho, \mathbf{w})|^{p} \mathrm{~d} \sigma(\mathbf{w})=\int_{\mathbb{T}^{d}} \mathrm{~d} \sigma(\mathbf{w}) \int_{\mathbb{T}^{1}}\left|\mathrm{M}\left(u_{\varrho \mathbf{w}}\right)(\omega)\right|^{p} d \omega \\
= & \int_{\mathbb{T}^{d}}\left\|\mathrm{M}\left(u_{\varrho \mathbf{w}}\right)\right\|_{p}^{p} \mathrm{~d} \sigma(\mathbf{w})=\int_{\mathbb{T}^{d}}\left\|u_{\varrho \mathbf{w}}\right\|_{p}^{p} \frac{\left\|\mathrm{M}\left(u_{\varrho \mathbf{w}}\right)\right\|_{p}^{p}}{\left\|u_{\varrho \mathbf{w}}\right\|_{p}^{p}} \mathrm{~d} \sigma(\mathbf{w}) \\
\leq & \max _{\mathbf{w} \in \mathbb{T}^{d}} \frac{\left\|\mathrm{M}\left(u_{\varrho \mathbf{w}}\right)\right\|_{p}^{p}}{\left\|u_{\varrho \mathbf{w}}\right\|_{p}^{p}} \int_{\mathbb{T}^{d}}\left\|u_{\varrho \mathbf{w}}\right\|_{p}^{p} \mathrm{~d} \sigma(\mathbf{w}) \\
\leq & \|\mathrm{M}\|_{L_{p}\left(\mathbb{T}^{1}\right) \rightarrow L_{p}\left(\mathbb{T}^{1}\right)} \int_{\mathbb{T}^{d}}\left\|u_{\varrho \mathbf{w}}\right\|_{p}^{p} \mathrm{~d} \sigma(\mathbf{w}) \\
= & \|\mathrm{M}\|_{L_{p}\left(\mathbb{T}^{1}\right) \rightarrow L_{p}\left(\mathbb{T}^{1}\right)} \int_{\mathbb{T}^{d}}|f(\varrho, \mathbf{w})|^{p} \mathrm{~d} \sigma(\mathbf{w}) .
\end{aligned}
$$

In the case $p=\infty$, we similarly obtain the estimate

$$
\begin{aligned}
& |\mathrm{M}(f)(\varrho, \omega \mathbf{w})|=\left|\mathrm{M}\left(u_{\varrho \mathbf{w}}\right)(\omega)\right| \\
= & \lim _{\rho \rightarrow 1-}\left|\mathrm{M}\left(u_{\varrho \mathbf{w}}\right)(\rho \omega)\right| \leq \max _{\omega \in \mathbb{T}^{1}}\left|\mathrm{M}\left(u_{\varrho \mathbf{w}}\right)(\omega)\right| \\
\leq & \|\mathrm{M}\|{ }_{L_{\infty, Y}\left(\mathbb{T}^{d}\right) \rightarrow L_{\infty, Y}\left(\mathbb{T}^{d}\right)} \max _{\omega \in \mathbb{T}^{1}}|f(\varrho, \omega \mathbf{w})| .
\end{aligned}
$$

From (3.7) and (3.8) in view of (3.5) it follows that for $1 \leq p \leq \infty$,

$$
\begin{aligned}
\|\mathrm{M}\|_{L_{p, Y}\left(\mathbb{T}^{d}\right) \rightarrow L_{p, Y}\left(\mathbb{T}^{d}\right)} & =\lim _{\varrho \rightarrow 1-} \sup _{f \in L_{p, Y}\left(\mathbb{T}^{d}\right)} \frac{\|\mathrm{M}(f)(\varrho, \cdot)\|_{p}}{\|f(\varrho, \cdot)\|_{p}} \\
& \leq\|\mathrm{M}\|_{L_{p}\left(\mathbb{T}^{1}\right) \rightarrow L_{p}\left(\mathbb{T}^{1}\right)} \cdot
\end{aligned}
$$

To prove the reverse inequality let us consider the continuation operator $Q$, given on $L_{p}\left(\mathbb{T}^{1}\right), 1 \leq p \leq \infty$, by the formula

$$
Q(g)\left(w_{1}, \mathbf{w}^{1}\right)=g\left(w_{1}\right),
$$

where $w_{1} \in \mathbb{T}^{1}, \mathbf{w}^{1}=\left(w_{2}, \ldots, w_{d}\right) \in \mathbb{T}^{d-1}$. 
It is easy to show that the continuation operator $Q$ is a linear isometry of the space $L_{p}\left(\mathbb{T}^{1}\right)$ in $L_{p}\left(\mathbb{T}^{d}\right)$. Therefore, taking into account the relation $Q(\mathrm{M}(f))=\mathrm{M}(Q(f))$, which is satisfied for any function $f \in L_{p}\left(\mathbb{T}^{1}\right)$, we obtain

$$
\begin{aligned}
\|\mathrm{M}(f)\|_{p} & =\|Q(\mathrm{M}(f))\|_{p}=\|\mathrm{M}(Q(f))\|_{p} \\
& \leq\|\mathrm{M}\|_{L_{p, Y}\left(\mathbb{T}^{d}\right) \rightarrow L_{p, Y}\left(\mathbb{T}^{d}\right)}\|Q(f)\|_{p} \\
& =\|\mathrm{M}\|_{L_{p, Y}\left(\mathbb{T}^{d}\right) \rightarrow L_{p, Y}\left(\mathbb{T}^{d}\right)}\|f\|_{p} .
\end{aligned}
$$

This implies the estimate

$$
\|\mathrm{M}\|_{L_{p}\left(\mathbb{T}^{1}\right) \rightarrow L_{p}\left(\mathbb{T}^{1}\right)} \leq\|\mathrm{M}\|_{L_{p, Y}\left(\mathbb{T}^{d}\right) \rightarrow L_{p, Y}\left(\mathbb{T}^{d}\right)},
$$

which in combination with (3.9) gives the relation (3.4).

\subsection{Auxiliary statements}

Let

$$
\mathcal{P}(\varrho, \mathbf{x}):=\prod_{j=1}^{d} \frac{1}{1-\varrho \mathrm{e}^{\mathrm{i} x_{j}}}+\prod_{j=1}^{d} \frac{1}{1-\varrho \mathrm{e}^{-i x_{j}}}-1 .
$$

Lemma 3.5. Assume that $f \in L_{1, Y}\left(\mathbb{T}^{d}\right), 0 \leq \varrho<1$ and $\mathbf{x} \in \mathbb{T}^{d}$. Then

$$
f(\varrho, \mathbf{x})=\int_{\mathbb{T}^{d}} f(\mathbf{x}+\mathbf{s}) \mathcal{P}(\varrho, \mathbf{s}) \mathrm{d} \sigma(\mathbf{s}) .
$$

Proof. By virtue of the definition of the set $L_{1, Y}\left(\mathbb{T}^{d}\right)$, we have

$$
f(\varrho, \mathbf{x})=\sum_{\nu=0}^{\infty} \varrho^{\nu} \sum_{\mathbf{k} \in Y:|\mathbf{k}|_{1}=\nu} \widehat{f}_{\mathbf{k}} \mathrm{e}_{\mathbf{k}}(\mathbf{x})
$$

On the other hand

$$
\begin{aligned}
\mathcal{P}(\varrho, \mathbf{x}) & =\sum_{k_{1}=0}^{\infty} \ldots \sum_{k_{d}=0}^{\infty} \varrho^{k_{1}+\ldots+k_{d}}\left(\mathrm{e}^{\mathrm{i}\left(k_{1} x_{1}+\ldots+k_{d} x_{d}\right)}+\mathrm{e}^{-\mathrm{i}\left(k_{1} x_{1}+\ldots+k_{d} x_{d}\right)}\right)-1 \\
& =1+\sum_{\nu=1}^{\infty} \varrho^{\nu} \sum_{\mathbf{k} \in Y:|\mathbf{k}|_{1}=\nu} \mathrm{e}_{\mathbf{k}}(\mathbf{x}) .
\end{aligned}
$$

Therefore, the right-hand side of (3.11) is equivalent to the right-hand side of (3.12).

Lemma 3.6. Assume that $f \in L_{p, Y}\left(\mathbb{T}^{d}\right), 1 \leq p \leq \infty, r=0,1, \ldots$ and $\varrho \in[0,1)$. Then the following relations are true:

$$
\left\|\frac{\partial^{r} f(\varrho, \cdot)}{\partial \varrho^{r}}\right\|_{p} \leq C_{1}(r) \frac{\|f\|_{p}}{(1-\varrho)^{r}}
$$

and

$$
\left\|A_{\varrho, r}^{[r]}(f)\right\|_{p} \leq C_{2}(r) \frac{\|f\|_{p}}{(1-\varrho)^{r}},
$$

where the constants $C_{1}(r)$ and $C_{2}(r)$ depend only on $r$. 
Proof. It is easy to see that the function $\partial^{r} f(\varrho, \mathbf{x}) / \partial \varrho^{r}$ can be considered as the image $\mathrm{M}_{1}(f)(\mathbf{x})$ of the multiplier generated by the sequence $\left\{\mu_{1, \nu}\right\}_{\nu=0}^{\infty}$, where $\mu_{1, \nu}=0$ for $\nu=0,1, \ldots, r-1$ and $\mu_{1, \nu}=\nu \cdot(\nu-1) \cdot \ldots \cdot(\nu-r+1) \varrho^{\nu-r}$ for $\nu \geq r$. Similarly, the function $A_{\varrho, r}^{[r]}(f)(\mathbf{x})$ can be considered as the image $\mathrm{M}_{2}(f)(\mathbf{x})$ of the multiplier generated by the sequence $\left\{\mu_{2, \nu}\right\}_{\nu=0}^{\infty}$ such that $\mu_{2, \nu}=0$ for $\nu=0,1, \ldots, r-1$ and $\mu_{2, \nu}=\nu ! \cdot \lambda_{\nu, r}(\varrho) /(\nu-r)$ ! for $\nu \geq r$. Therefore, to prove estimates (3.14) and $(3.15)$ it is sufficient to apply Lemma 3.4 and the estimates (23) and (22) for the norms of the corresponding multipliers in the space $L_{p}\left(\mathbb{T}^{1}\right)$ from [13].

For any $f \in L_{p}\left(\mathbb{T}^{d}\right), 1 \leq p \leq \infty, 0 \leq \varrho<1$ and $r=0,1,2, \ldots$, we set

$$
M_{p}(\varrho, f, r):=\varrho^{r}\left\|\frac{\partial^{r} f(\varrho, \cdot)}{\partial \varrho^{r}}\right\|_{p}=\left\|(f(\varrho, \cdot))^{[r]}\right\|_{p}
$$

Lemma 3.7. Assume that $f \in L_{p, Y}\left(\mathbb{T}^{d}\right), 1 \leq p \leq \infty$. Then for any numbers $n \in \mathbb{N}$ and $\varrho \in[0,1)$,

$$
\begin{aligned}
& C_{3}(n)(1-\varrho)^{n} M_{p}(\varrho, f, n) \leq K_{n}(1-\varrho, f)_{p} \\
\leq & C_{4}(n)\left(\left\|f-A_{\varrho, n}(f)\right\|_{p}+(1-\varrho)^{n} M_{p}(\varrho, f, n)\right),
\end{aligned}
$$

where the constants $C_{3}(n)$ and $C_{4}(n)$ depend only on $n$.

Proof. First, let us note that the statement of Lemma 3.7 is trivial in the case, when $f$ is a polynomial of the form

$$
f(\mathbf{x})=\sum_{\nu=0}^{n-1} \sum_{|\mathbf{k}|_{1}=\nu} \widehat{f}_{\mathbf{k}} \mathrm{e}_{\mathbf{k}}(\mathbf{x}),
$$

as well as in the case, when $\varrho=0$. Therefore, further in the proof, we exclude these two cases.

Let $g$ be a function such that $g^{[n]} \in L_{p}\left(\mathbb{T}^{d}\right)$. Using Lemma 3.6, we get

$$
\begin{aligned}
\left\|\frac{\partial^{n} f(\varrho, \cdot)}{\partial \varrho^{n}}\right\|_{p} & =\left\|\frac{\partial^{n}(f-g)(\varrho, \cdot)}{\partial \varrho^{n}}+\frac{\partial^{n} g(\varrho, \cdot)}{\partial \varrho^{n}}\right\|_{p} \\
& \leq C_{1}(n) \frac{\|f-g\|_{p}}{(1-\varrho)^{n}}+\left\|\frac{\partial^{n} g(\varrho, \cdot)}{\partial \varrho^{n}}\right\|_{p}
\end{aligned}
$$

Setting $C_{3}(n)=\min \left\{1,1 / C_{1}(n)\right\}$ and taking into account relations (3.1), (3.16) and the inequality $\left\|g^{[n]}(\varrho, \cdot)\right\|_{p} \leq\left\|g^{[n]}\right\|_{p}$, we see that

$$
C_{3}(n)(1-\varrho)^{n} M_{p}(\varrho, f, n) \leq\|f-g\|_{p}+(1-\varrho)^{n}\left\|g^{[n]}\right\|_{p} .
$$

Considering the infimum over all functions $g$ such that $g^{[n]} \in L_{p}\left(\mathbb{T}^{d}\right)$, we conclude that

$$
C_{3}(n)(1-\varrho)^{n} M_{p}(\varrho, f, n) \leq K_{n}(1-\varrho, f)_{p} .
$$

On the other hand, from the definition of the $K$-functional, it follows that

$$
K_{n}(1-\varrho, f)_{p} \leq\left\|f-A_{\varrho, n}(f)\right\|_{p}+(1-\varrho)^{n}\left\|A_{\varrho, n}^{[n]}(f)\right\|_{p} .
$$


According to (2.7) and (3.1), we have

$$
\begin{aligned}
A_{\varrho, n}^{[n]}(f)(\mathbf{x}) & =\left(\sum_{k=0}^{n-1} \frac{(f(\varrho, \cdot))^{[k]}(\cdot)}{\varrho^{k} k !}(1-\varrho)^{k}\right)^{[n]}(\mathbf{x}) \\
& =\sum_{k=0}^{n-1} \frac{\left((f(\varrho, \cdot))^{[k]}(\cdot)\right)^{[n]}(\mathbf{x})}{\varrho^{k} k !}(1-\varrho)^{k} .
\end{aligned}
$$

Since for any nonnegative integers $k$ and $n$

$$
\left((f(\varrho, \cdot))^{[n]}(\cdot)\right)^{[k]}(\mathbf{x})=\left((f(\varrho, \cdot))^{[k]}(\cdot)\right)^{[n]}(\mathbf{x}),
$$

we obtain

$$
A_{\varrho, n}^{[n]}(f)(\mathbf{x})=\sum_{k=0}^{n-1} \frac{\left((f(\varrho, \cdot))^{[n]}(\cdot)\right)^{[k]}(\mathbf{x})}{\varrho^{k} k !}(1-\varrho)^{k}
$$

This yields

$$
\left\|A_{\varrho, n}^{[n]}(f)\right\|_{p} \leq \sum_{k=0}^{n-1} \frac{\left\|\left((f(\varrho, \cdot))^{[n]}(\cdot)\right)^{[k]}\right\|_{p}}{\varrho^{k} k !}(1-\varrho)^{k},
$$

where by virtue of Lemma 3.6 and (3.16)

$$
\left\|\left((f(\varrho, \cdot))^{[n]}(\cdot)\right)^{[k]}\right\|_{p} \leq M_{p}(\varrho, f, n) \frac{C_{1}(k) \varrho^{k}}{(1-\varrho)^{k}} .
$$

Therefore,

$$
\left\|A_{\varrho, n}^{[n]}(f)\right\|_{p} \leq M_{p}(\varrho, f, n) \sum_{k=0}^{n-1} \frac{C_{1}(k)}{k !} .
$$

Setting

$$
C_{4}(n)=\max \left\{1, \sum_{k=0}^{n-1} C_{1}(k) / k !\right\}
$$

and combining relations (3.18) and (3.22), we obtain the right-hand inequality in (3.17).

Lemma 3.8. Assume that $f \in L_{p}\left(\mathbb{T}^{d}\right), 1 \leq p \leq \infty, 0 \leq \varrho<1$ and $r=2,3, \ldots$ such that

$$
\int_{\varrho}^{1}\left\|\frac{\partial^{r} f(\zeta, \cdot)}{\partial \zeta^{r}}\right\|_{p}(1-\zeta)^{r-1} d \zeta<\infty
$$

Then for almost all $\mathbf{x} \in \mathbb{T}^{d}$,

$$
f(\mathbf{x})-A_{\varrho, r}(f)(\mathbf{x})=\frac{1}{(r-1) !} \int_{\varrho}^{1} \frac{\partial^{r} f(\zeta, \mathbf{x})}{\partial \zeta^{r}}(1-\zeta)^{r-1} d \zeta .
$$

Proof. For fixed $r=2,3, \ldots$ and $0 \leq \varrho<1$, the integral on the right-hand side of (3.24) defines a certain function $F(\mathbf{x})$. By virtue of (3.23) and the integral Minkowski inequality, we conclude that the function $F$ belongs to the space $L_{p}\left(\mathbb{T}^{d}\right)$. Let us find the Fourier coefficients of $F$ and compare them with the Fourier coefficients of the function $G:=f-A_{\varrho, r}(f)$. 
Since for any $\nu=r, r+1 \ldots$,

$$
\frac{1}{(r-1) ! \cdot(\nu-r) !} \int_{\varrho}^{\varrho_{1}} \zeta^{\nu-r}(1-\zeta)^{r-1} d \zeta=\sum_{j=0}^{r-1} \frac{\varrho_{1}^{\nu-j}\left(1-\varrho_{1}\right)^{j}-\varrho^{\nu-j}(1-\varrho)^{j}}{j ! \cdot(\nu-j) !}
$$

then in view of $(2.9)$ for a fixed $\varrho_{1} \in(\varrho, 1)$, we have

$$
\begin{aligned}
& \frac{1}{(r-1) !} \int_{\varrho}^{\varrho_{1}} \frac{\partial^{r} f(\zeta, \mathbf{x})}{\partial \zeta^{r}}(1-\zeta)^{r-1} \mathrm{~d} \zeta \\
= & \sum_{\nu=r}^{\infty} \sum_{|\mathbf{k}|_{1}=\nu} \frac{\nu ! \cdot \widehat{f}_{\mathbf{k}} \cdot \mathrm{e}_{\mathbf{k}}(\mathbf{x})}{(r-1) ! \cdot(\nu-r) !} \int_{\varrho}^{\varrho_{1}} \zeta^{\nu-r}(1-\zeta)^{r-1} \mathrm{~d} \zeta \\
= & \sum_{\nu=r}^{\infty} \sum_{|\mathbf{k}|_{1}=\nu} \widehat{f}_{\mathbf{k}} \mathrm{e}_{\mathbf{k}}(\mathbf{x}) \sum_{j=0}^{r-1}\left(\begin{array}{l}
\nu \\
j
\end{array}\right)\left(\varrho_{1}^{\nu-j}\left(1-\varrho_{1}\right)^{j}-\varrho^{\nu-j}(1-\varrho)^{j}\right) .
\end{aligned}
$$

Now if in relation $(3.25)$, the value $\varrho_{1}$ tends to $1-$, then we see that the Fourier coefficients $\widehat{F}_{\mathbf{k}}$ of the function $F$ are equivalent to zero when $|\mathbf{k}|_{1}=\nu<r$ and for $|\mathbf{k}|_{1} \geq r$

$$
\widehat{F}_{\mathbf{k}}=\widehat{f}_{\mathbf{k}} \cdot\left(1-\sum_{j=0}^{r-1}\left(\begin{array}{l}
\nu \\
j
\end{array}\right)(1-\varrho)^{j} \varrho^{\nu-j}\right)=\left(1-\lambda_{\nu, r}(\varrho)\right) \widehat{f}_{\mathbf{k}} .
$$

Therefore, for all $\mathbf{k} \in \mathbb{Z}^{d}$ we have $\widehat{F}_{\mathbf{k}}=\left(1-\lambda_{\nu, r}(\varrho)\right) \widehat{f}_{\mathbf{k}}=\widehat{G}_{\mathbf{k}}$. Hence, for almost all $\mathbf{x} \in \mathbb{T}^{d}$, relation $(3.24)$ holds.

\subsection{Proof of main results}

Proof of Theorem 3.1. Assume that the function $f$ is such that $f^{[r-n]} \in L_{p, Y}\left(\mathbb{T}^{d}\right)$ and relation (3.2) is satisfied. Let us apply the first inequality of Lemma 3.7 to the function $f^{[r-n]}$. In view of (3.1) and (3.16), we obtain

$$
C_{3}(n)(1-\varrho)^{n} M_{p}(\varrho, f, r) \leq K_{n}\left(1-\varrho, f^{[r-n]}\right)_{p} .
$$

This yields

$$
M_{p}(\varrho, f, r)=\mathcal{O}(1)(1-\varrho)^{-n} \omega(1-\varrho), \quad \varrho \rightarrow 1-.
$$

Using relations $(3.16),(3.27),(\mathcal{Z})$ and the integral Minkowski inequality, we obtain

$$
\begin{array}{r}
\int_{\varrho}^{1}\left\|\frac{\partial^{r} f(\zeta, \cdot)}{\partial \zeta^{r}}\right\|_{p}(1-\zeta)^{r-1} \mathrm{~d} \zeta \leq \int_{\varrho}^{1} M_{p}(\zeta, f, r) \frac{(1-\zeta)^{r-1}}{\zeta^{r}} \mathrm{~d} \zeta \\
\leq C_{1}(1-\varrho)^{r-n} \int_{\varrho}^{1} \frac{\omega(1-\zeta)}{1-\zeta} \mathrm{d} \zeta=\mathcal{O}\left((1-\varrho)^{r-n} \omega(1-\varrho)\right), \varrho \rightarrow 1-.
\end{array}
$$

Therefore, for almost all $\mathbf{x} \in \mathbb{T}^{d}$, relation (3.24) holds. Hence, by virtue of (3.24), using the integral Minkowski inequality and (3.28), we finally get (3.3):

$$
\begin{gathered}
\left\|f-A_{\varrho, r}(f)\right\|_{p} \leq \frac{1}{(r-1) !} \int_{\varrho}^{1} M_{p}(\zeta, f, r) \frac{(1-\zeta)^{r-1}}{\zeta^{r}} \mathrm{~d} \zeta \\
=\mathcal{O}\left((1-\varrho)^{r-n} \omega(1-\varrho)\right), \quad \varrho \rightarrow 1-.
\end{gathered}
$$


Proof of Theorem 3.2. First, let us note that for any function $f \in L_{p}\left(\mathbb{T}^{d}\right)$ and all fixed numbers $s, r \in \mathbb{N}$ and $\varrho \in(0,1)$, we have

$$
\begin{aligned}
\left\|A_{\varrho, r}^{[s]}(f)\right\|_{p} & =\left\|\sum_{\nu=s}^{\infty} \frac{\nu !}{(\nu-s) !} \lambda_{\nu, r}(\varrho) \sum_{|\mathbf{k}|_{1}=\nu} \widehat{f}_{\mathbf{k}} \mathrm{e}_{\mathbf{k}}\right\|_{p} \\
& \leq 2 r\|f\|_{p}\left(\sum_{\nu=s}^{\max \{s, r\}-1} \frac{\nu !}{(\nu-s) !}+\sum_{\nu \geq \max \{s, r\}} q^{\nu} \nu^{s+r-1}\right)<\infty,
\end{aligned}
$$

where $0<q=\max \{1-\varrho, \varrho\}<1$. In the case where $s \geq r$, the sum $\sum_{\nu=s}^{s-1}$ is set equal to zero. Put $\varrho_{k}:=1-2^{-k}, k \in \mathbb{N}$, and $A_{k}:=A_{k}(f):=A_{\varrho_{k}, r}(f)$. For any $\mathbf{x} \in \mathbb{T}^{d}$ and $s \in \mathbb{N}$, consider the series

$$
A_{0}^{[s]}(f)(\mathbf{x})+\sum_{k=1}^{\infty}\left(A_{k}^{[s]}(f)(\mathbf{x})-A_{k-1}^{[s]}(f)(\mathbf{x})\right) .
$$

According to the definition of the operator $A_{\varrho, r}$, we see that for any $\varrho_{1}, \varrho_{2} \in[0,1)$ and $r \in \mathbb{N}$,

$$
A_{\varrho_{1}, r}\left(A_{\varrho_{2}, r}(f)\right)=A_{\varrho_{2}, r}\left(A_{\varrho_{1}, r}(f)\right) .
$$

By virtue of Lemma 3.6 and relation (3.3), for any $k \in \mathbb{N}$ and $s \in \mathbb{N}$, we have

$$
\begin{aligned}
& \left\|A_{k}^{[s]}-A_{k-1}^{[s]}\right\|_{p}=\left\|A_{k}^{[s]}\left(f-A_{k-1}(f)\right)-A_{k-1}^{[s]}\left(f-A_{k}(f)\right)\right\|_{p} \\
\leq & \left\|A_{k}^{[s]}\left(f-A_{k-1}(f)\right)\right\|_{p}+\left\|A_{k-1}^{[s]}\left(f-A_{k}(f)\right)\right\|_{p} \\
\leq & C_{2}(s) \frac{\left\|f-A_{k-1}(f)\right\|_{p}}{\left(1-\varrho_{k}\right)^{s}}+C_{2}(s) \frac{\left\|f-A_{k}(f)\right\|_{p}}{\left(1-\varrho_{k-1}\right)^{s}} \\
= & \mathcal{O}\left(\frac{\omega\left(1-\varrho_{k-1}\right)}{\left(1-\varrho_{k}\right)^{s-r+n}}\right)+\mathcal{O}\left(\frac{\omega\left(1-\varrho_{k}\right)}{\left(1-\varrho_{k-1}\right)^{s-r+n}}\right), \quad k \rightarrow \infty .
\end{aligned}
$$

Therefore, for any $s \leq r-n$,

$$
\left\|A_{k}^{[s]}-A_{k-1}^{[s]}\right\|_{p}=\mathcal{O}\left(\omega\left(1-\varrho_{k-1}\right)\right)=\mathcal{O}\left(\omega\left(2^{-(k-1)}\right)\right), \quad k \rightarrow \infty .
$$

Consider the sum $\sum_{k=1}^{N} \omega\left(2^{1-k}\right), N \in \mathbb{N}$. Taking into account the monotonicity of the function $\omega$ and $(\mathcal{Z})$, we see that for all $N \in \mathbb{N}$,

$$
\sum_{k=1}^{N} \omega\left(2^{1-k}\right) \leq \omega(1)+\int_{1}^{N} \omega\left(2^{1-t}\right) \mathrm{d} t=\omega(1)+\int_{2^{1-N}}^{1} \frac{\omega(\tau) d \tau}{\tau \ln 2}<\infty .
$$

Combining the last relation and (3.31), we conclude that the series in (3.29) converges in the norm of the space $L_{p}\left(\mathbb{T}^{d}\right), 1 \leq p \leq \infty$. Hence, by virtue of the Banach-Alaoglu 
theorem, for any $s=0,1, \ldots, r-n$, there exists the subsequence

$$
S_{N_{j}}^{[s]}(\mathbf{x})=A_{0}^{[s]}(f)(\mathbf{x})+\sum_{k=1}^{N_{j}}\left(A_{k}^{[s]}(f)(\mathbf{x})-A_{k-1}^{[s]}(f)(\mathbf{x})\right), \quad j=1,2, \ldots
$$

of partial sums of this series, converging to a certain function $g \in L_{p}\left(\mathbb{T}^{d}\right)$ almost everywhere on $\mathbb{T}^{d}$ as $j \rightarrow \infty$.

Let us show that $g=f^{[s]}$. For this, let us find the Fourier coefficients of the function $g$. For any fixed $\mathbf{k} \in \mathbb{Z}^{d}$ and all $j=1,2, \ldots$, we have

$$
\widehat{g}_{\mathbf{k}}:=\int_{\mathbb{T}^{d}} S_{N_{j}}^{[s]}(\mathbf{x}) \overline{\mathrm{e}}_{\mathbf{k}}(\mathbf{x}) \mathrm{d} \sigma(\mathbf{x})+\int_{\mathbb{T}^{d}}\left(g(\mathbf{x})-S_{N_{j}}^{[s]}(\mathbf{x})\right) \overline{\mathrm{e}}_{\mathbf{k}}(\mathbf{x}) \mathrm{d} \sigma(\mathbf{x}) .
$$

Since the sequence $\left\{S_{N_{j}}^{[s]}\right\}_{j=1}^{\infty}$ converges almost everywhere on $\mathbb{T}^{d}$ to the function $g$, the second integral on the right-hand side of the last equality tends to zero as $j \rightarrow \infty$. By virtue of (3.32) and the definition of the radial derivative, for $|\mathbf{k}|_{1}=\nu<s$ the first integral is equal to zero, and for all $|\mathbf{k}|_{1}=\nu \geq s$,

$$
\int_{\mathbb{T}^{d}} S_{N_{j}}^{[s]}(\mathbf{x}) \overline{\mathrm{e}}_{\mathbf{k}}(\mathbf{x}) \mathrm{d} \sigma(\mathbf{x})=\lambda_{\nu, r}\left(1-2^{-N_{j}}\right) \frac{\nu !}{(\nu-s) !} \widehat{f}_{\mathbf{k}} \underset{j \rightarrow \infty}{\longrightarrow} \frac{\nu !}{(\nu-s) !} \widehat{f}_{\mathbf{k}} .
$$

Therefore, the equality $g=f^{[s]}$ is true.

Hence, for the function $f$ and all $s=0,1, \ldots, r-n$, there exists the derivative $f^{[s]}$ and $f^{[s]} \in L_{p}\left(\mathbb{T}^{d}\right)$.

Now, let us prove the estimate (3.27). By virtue of (3.16), (3.30), for any $k \in \mathbb{N}$ and $\varrho \in(0,1)$, we have

$$
\begin{aligned}
& M_{p}\left(\varrho, A_{k}-A_{k-1}, r\right) \leq\left\|A_{k}^{[r]}-A_{k-1}^{[r]}\right\|_{p} \\
& =\mathcal{O}\left(\frac{\omega\left(1-\varrho_{k-1}\right)}{\left(1-\varrho_{k}\right)^{n}}\right)+\mathcal{O}\left(\frac{\omega\left(1-\varrho_{k}\right)}{\left(1-\varrho_{k-1}\right)^{n}}\right) \\
& =\mathcal{O}\left(2^{k n} \omega\left(2^{-k+1}\right)+2^{(k-1) n} \omega\left(2^{-k}\right)\right) \\
& =\mathcal{O}\left(2^{(k-1) n} \omega\left(2^{-(k-1)}\right)\right), \quad k \rightarrow \infty .
\end{aligned}
$$

By virtue of (3.16), (3.14) and (3.3), for any $r \in \mathbb{N}$ and $\varrho \in(0,1)$, we obtain

$$
M_{p}\left(\varrho, f-A_{\varrho, r}(f), r\right)=\mathcal{O}(1) \frac{\left\|f-A_{\varrho, r}(f)\right\|_{p}}{(1-\varrho)^{r}}=\mathcal{O}\left(\frac{\omega(1-\varrho)}{(1-\varrho)^{n}}\right), \quad \varrho \rightarrow 1-.
$$

Therefore, for $N \rightarrow \infty$,

$$
M_{p}\left(\varrho_{N}, f-A_{N}(f), r\right)=\mathcal{O}\left(\frac{\omega\left(1-\varrho_{N}\right)}{\left(1-\varrho_{N}\right)^{n}}\right)=\mathcal{O}\left(2^{N n} \omega\left(2^{-N}\right)\right)
$$

Consider the sum $\sum_{k=1}^{N} 2^{(k-1) n} \omega\left(2^{-(k-1)}\right), N \in \mathbb{N}$. Since the function $\omega$ satisfies the condition $\left(\mathcal{Z}_{n}\right)$, the function $\omega(t) / t^{n}$ almost decreases on $(0,1]$, i.e., there exists the 
number $C>0$ such that $\omega\left(t_{1}\right) / t_{1}^{n} \geq C \omega\left(t_{2}\right) / t_{2}^{n}$ for any $0<t_{1}<t_{2} \leq 1$ (see, for example [1]). Therefore,

$$
\begin{aligned}
& \sum_{k=1}^{N} 2^{(k-1) n} \omega\left(2^{-(k-1)}\right) \\
\leq & C\left(2^{(N-1) n} \omega\left(2^{-(N-1)}\right)+\int_{1}^{N} 2^{(t-1) n} \omega\left(2^{-(t-1)}\right) \mathrm{d} t\right) \\
\leq & C\left(2^{(N-1) n} \omega\left(2^{-(N-1)}\right)+\int_{2^{-N+1}}^{1} \frac{\omega(\tau) d \tau}{\tau^{n+1} \ln 2}\right) \\
= & \mathcal{O}\left(2^{(N-1) n} \omega\left(2^{-(N-1)}\right)\right)=\mathcal{O}\left(2^{N n} \omega\left(2^{-N}\right)\right), \quad N \rightarrow \infty .
\end{aligned}
$$

Putting $\varrho=\varrho_{N}$ and taking into account relations (3.33), (3.34), (3.35) and

$$
A_{0}(\mathbf{x})=S_{r-1}(f)(\mathbf{x})=\sum_{|\mathbf{k}|_{1} \leq r-1} \widehat{f}_{\mathbf{k}} \mathrm{e}_{\mathbf{k}}(\mathbf{x}),
$$

we get

$$
\begin{aligned}
& M_{p}\left(\varrho_{N}, f, r\right)=M_{p}\left(\varrho_{N}, f-S_{r-1}(f), r\right) \\
= & M_{p}\left(\varrho_{N}, f-A_{\varrho_{N}}+\sum_{k=1}^{N}\left(A_{k}-A_{k-1}\right), r\right)=\mathcal{O}\left(\sum_{k=1}^{N} 2^{(k-1) n} \omega\left(2^{-(k-1)}\right)\right) \\
= & \mathcal{O}\left(2^{N n} \omega\left(2^{-N}\right)\right)=\mathcal{O}\left(\left(1-\varrho_{N}\right)^{-n} \omega\left(1-\varrho_{N}\right)\right), \quad N \rightarrow \infty
\end{aligned}
$$

If the function $\omega$ satisfies the condition $\left(\mathcal{Z}_{n}\right)$, then $\sup _{t \in[0,1]}(\omega(2 t) / \omega(t))<\infty$ (see, for example [1]). Furthermore, for all $\varrho \in\left[\varrho_{N-1}, \varrho_{N}\right]$, we have $1-\varrho_{N} \leq 1-\varrho \leq 2\left(1-\varrho_{N}\right)$. Hence, relation (3.36) yields the estimate (3.27).

Now, applying the second inequality in Lemma 3.7 to the function $f^{[r-n]}$, we get

$$
\begin{aligned}
K_{n}\left(1-\varrho, f^{[r-n]}\right)_{p} & \leq C_{4}(n)\left(\left\|f^{[r-n]}-A_{\varrho, n}\left(f^{[r-n]}\right)\right\|_{p}\right. \\
& \left.+(1-\varrho)^{n} M_{p}(\varrho, f, r)\right) .
\end{aligned}
$$

By virtue of (3.16) and (3.27), we see that for $\varrho \in[1 / 2,1)$,

$$
\begin{aligned}
& \int_{\varrho}^{1}\left\|\frac{\partial^{n} f^{[r-n]}(\zeta, \cdot)}{\partial \zeta^{n}}\right\|_{p}(1-\zeta)^{n-1} \mathrm{~d} \zeta \\
= & \int_{\varrho}^{1}\left\|(f(\zeta, \cdot))^{[r]}\right\|_{p} \frac{(1-\zeta)^{n-1}}{\zeta^{n}} \mathrm{~d} \zeta \\
= & \int_{\varrho}^{1} M_{p}(\zeta, f, r) \frac{(1-\zeta)^{n-1}}{\zeta^{n}} \mathrm{~d} \zeta \\
\leq & C_{1} \int_{\varrho}^{1} \frac{\omega(1-\zeta)}{1-\zeta} \mathrm{d} \zeta=\mathcal{O}(\omega(1-\varrho)), \quad \varrho \rightarrow 1-.
\end{aligned}
$$


Therefore, we can apply Lemma 3.8 to the function $f^{[r-n]}$. Taking into account (3.16), we obtain

$$
f^{[r-n]}(\mathbf{x})-A_{\varrho, n}\left(f^{[r-n]}\right)(\mathbf{x})=\frac{1}{(n-1) !} \int_{\varrho}^{1}(f(\zeta, \cdot))^{[r]}(\mathbf{x}) \frac{(1-\zeta)^{n-1}}{\zeta^{n}} \mathrm{~d} \zeta .
$$

Using the integral Minkowski inequality and (3.38), we conclude

$$
\begin{aligned}
\left\|f^{[r-n]}-A_{\varrho, n}\left(f^{[r-n]}\right)\right\|_{p} & \leq \frac{1}{(n-1) !} \int_{\varrho}^{1} M_{p}(\zeta, f, r) \frac{(1-\zeta)^{n-1}}{\zeta^{n}} \mathrm{~d} \zeta \\
& =\mathcal{O}(\omega(1-\varrho)), \quad \varrho \rightarrow 1-.
\end{aligned}
$$

Combining relations (3.37), (3.27) and (3.39), we finally get (3.2).

Acknowledgments. This work is partially supported by the Grant H2020-MSCARISE-2014, project number 645672 (AMMODIT: Approximation Methods for Molecular Modelling and Diagnosis Tools) and the Grant of the NAS of Ukraine to research laboratories / groups of young scientists of the NAS of Ukraine for conducting researches on priority directions of science and technology development in 2018, project number 16-10/2018.

\section{References}

[1] Bari, N.K., Stechkin, S.B., Best approximations and differential properties of two conjugate functions, (Russian), Tr. Mosk. Mat. Obshch., 5(1956), 483-522.

[2] Butzer, P.L., Beziehungen zwischen den Riemannschen, Taylorschen und gewöhnlichen Ableitungen reellwertiger Funktionen, Math. Ann., 144(1961), 275-298.

[3] Butzer, P.L., Nessel, R., Fourier Analysis and Approximation, One-Dimensional Theory, Basel, New York, 1971.

[4] Butzer, P.L., Sunouchi, G., Approximation theorems for the solution of Fourier's problem and Dirichlet's problem, Math. Ann., 155(1964), 316-330.

[5] Butzer, P.L., Tillmann, H.G., Approximation theorems for semi-groups of bounded linear transformations, Math. Ann., 140(1960), 256-262.

[6] Chandra, P., Mohapatra, R.N., Approximation of functions by $\left(J, q_{n}\right)$ means of Fourier series, Approx. Theory Appl., 4(1988), no. 2, 49-54.

[7] Chui, C.K., Holland, A.S.B., On the order of approximation by Euler and Taylor means, J. Approx. Theory, 39(1983), no. 1, 24-38.

[8] DeVore, R.A., Lorentz, G.G. Constructive Approximation, Berlin, Springer Verlag, 1993.

[9] Edwards, R.E., Fourier Series: a Modern Introduction, Vol. 2, New York, NY, Springer New York, 1982.

[10] Holland, A.S.B., Sahney, B.N., Mohapatra, R.N., $L_{p}$ approximation of functions by Euler means, Rend. Mat. Appl., VII(3)(1983), no. 2, 341-355.

[11] Leis, R., Approximationssätze für stetige Operatoren, Arch. Math., 14(1963), 120-129.

[12] Mohapatra, R.N., Holland, A.S.B., Sahney, B.N., Functions of class $\operatorname{Lip}(\alpha, p)$ and their Taylor mean, J. Approx. Theory, 45(1985), no. 4, 363-374.

[13] Prestin, J., Savchuk, V.V., Shidlich, A.L., Direct and inverse approximation theorems of $2 \pi$-periodic functions by Taylor-Abel-Poisson means, Ukr. Mat. Journ., 69(2017), no. $5,766-781$. 
[14] Rudin, W., Function Theory in Polydiscs, W.A. Benjamin Inc., New York, Amsterdam, 1969.

[15] Savchuk, V.V., Approximation of holomorphic functions by Taylor-Abel-Poisson means, Ukr. Mat. Journ., 59(2007), no. 9, 1397-1407.

[16] Savchuk, V.V., Savchuk, M.V., Norms of multipliers and best approximations of holomorphic multivariable functions, Ukr. Mat. Journ., 54(2002), no. 12, 2025-2037.

[17] Savchuk, V.V., Shidlich, A.L., Approximation of functions of several variables by linear methods in the space $S^{p}$, Acta Sci. Math. (Szeged), 80(2014), no. 3-4, 477-489.

[18] Trigub, R.M., Belinsky, E.S., Fourier analysis and approximation of functions, Dordrecht, Kluwer Academic Publishers, 2004.

[19] Zastavnyi, V.P., Savchuk, V.V., Approximation of classes of convolutions by linear operators of a special form, Math. Notes, 90(2011), no. 3, 333-343.

Jürgen Prestin

University of Lübeck

Institute of Mathematics

Ratzeburger Allee 160

23562 Lübeck, Germany

e-mail: prestin@math.uni-luebeck.de

Viktor Savchuk

Institute of Mathematics of the National Academy of Sciences of Ukraine

3, Tereschenkivska Street

01004 Kyiv, Ukraine

e-mail: vicsavchuk@gmail.com

Andrii Shidlich

Institute of Mathematics of the National Academy of Sciences of Ukraine

3, Tereschenkivska Street

01004 Kyiv, Ukraine

e-mail: shidlich@gmail.com 